STUDY OF THE MAJOR CONDITIONS INFLUENCING STEP HEIGHT MEASUREMENT USING INTERFERENCE MICROSCOPE

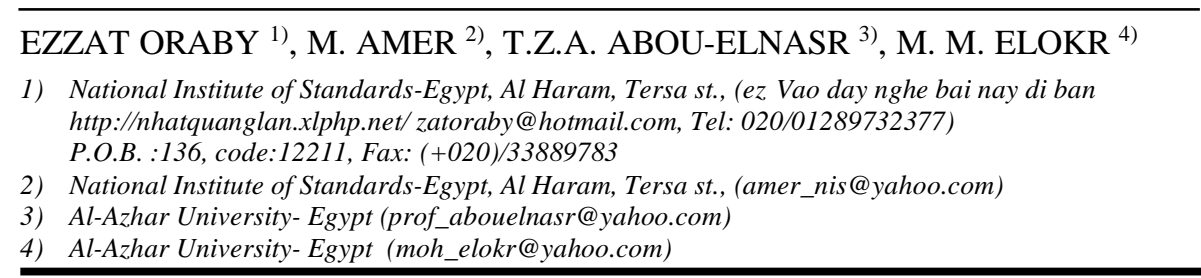

\begin{abstract}
Step height measurements in micro and nano scale are essential to the micro-technology. The interferometric measurement is carried out using two main techniques: white light scanning interferometry (WLI) and phase stepping interferometry (PSI). A step height standard sample of nominal step height value $\mathrm{h}$ of $90.1 \mathrm{~nm}$ has been studied using the PSI mode with an optical filter light of $\lambda=633.4 \mathrm{~nm}$ against three parameters: vertical distance (z) between objective and sample, intensity of the incident light and the inclination angles of the sample to the incident light. The effect of numerical aperture of objectives has been referenced to some literature. The total budget of studied parameters for the sample $90.1 \mathrm{~nm}$ leads to expanded uncertainty of $0.46 \mathrm{~nm}$.
\end{abstract}

\title{
Introduction
}

Step height measurements of thin films are important in the field of development of high-precision to enhance a special function of, and better performance for coated devices which are widely used in the optics, semiconductor, nanometric technologies and materials industries. [1-4]

Such measurements of the ever-smaller features on semiconductor chips require surface profilers with high vertical and lateral resolution are carried out by different techniques: stylus methods, optical methods and recently scanned probe microscopes (SPM) such as the scanning tunneling microscope (STM) and atomic force microscope (AFM). To calibrate the height scale of these instruments lor to compare the performance of these instruments, step-height standards are used with the appropriate length scale for each beginning with micrometers, nanometers and to sub angstrom respectively. [5]

Two sub-techniques are followed in Interference microscopy: WLI and PSI, although former eliminates the $2 \pi$ ambiguity introduced by the latter but the latter keeps offering a relatively long coherence and good accuracy [6].

Phase measurements are real-time noncontact extremely useful for testing highprecision optical systems. A wide range of physical parameters such as stress, vibration, displacement, height, and surface profile can be obtained by using PSI.

\section{Interference Microscope:}

An extended broadband light source is imaged at the pupil of a microscope objective equipped with a Mirau interferometer. The reference mirror position is adjusted so that high-contrast optical interference fringes are observed at the camera when the sample surface is at best-focus. The complete measurement is performed by moving the stage with respect to objective and interferometer vertically while recording the intensity pattern at each pixel of the camera. [6]. 


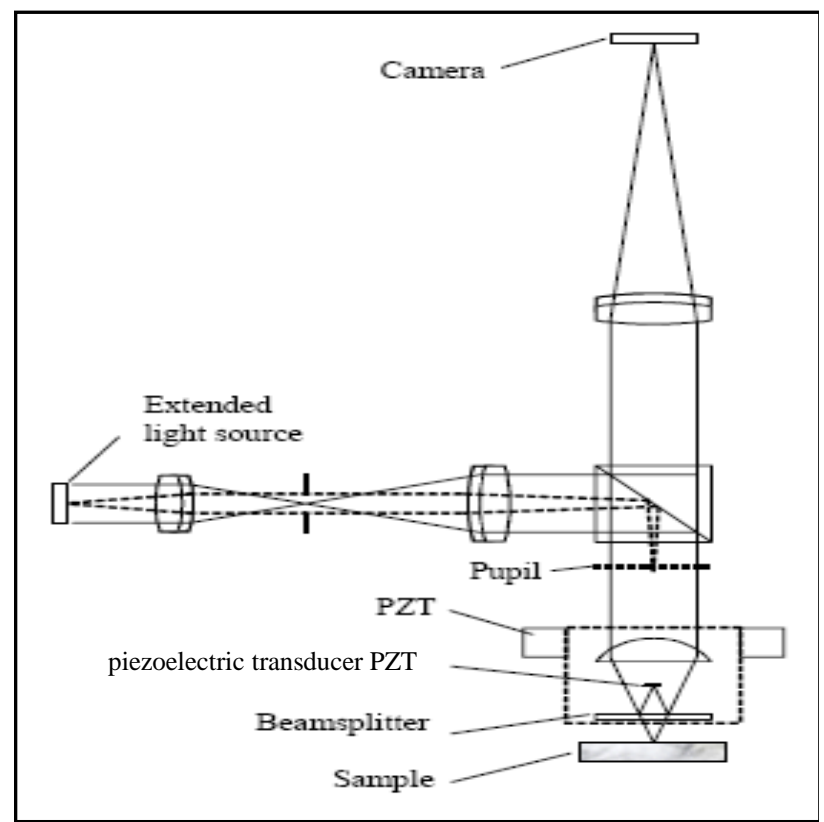

Fig.(1) a diagram for the Mirau type interference microscope

In the testing of optical components and optical systems there are many requirements on the precision and accuracy, measurement time, ease of use, dynamic range, and environmental conditions. Interferometry, and in particular phase shifting interferometry, has the precision acceptable. [7]

Phase stepping interferometry (PSI)

A height measurement with 1- $\AA$ resolution can be achieved. By measuring the intensity of the interference signal as the phase is shifted, we can obtain the phase. All methods of phase extraction assume knowledge of the relationship between the intensity of the interference signal and its phase. In the usual case, assuming monochromatic plane waves, the interference signal intensity varies sinusoidally with the phase.

Phase stepping algorithm equations:

Mathematically, the intensity distribution I(u, v) captured by the camera during an acquisition of a single image is a series of interferograms with a certain phase increment between them is obtained. Once these phase-shifted patterns have been combined using a phase-shifting algorithm, we can obtain the phase values modulo $2 \pi$ for all of the full field of view simultaneously. This method can provide as accuracy level of up to $\lambda / 1000$ if the ambient conditions and experimental setup are well controlled $[3,6]$

$I(u, v)=I_{b}(u, v)+I_{m}(u, v) \cos [\Delta \varphi(u, v)]$

where $\mathrm{I}_{\mathrm{b}}(\mathrm{u}, \mathrm{v})$ and $\mathrm{I}_{\mathrm{m}}(\mathrm{u}, \mathrm{v})$ are the background and modulation intensities, respectively, and $\Delta \varphi(\mathrm{u}, \mathrm{v})$ is the interference phase information at position $(\mathrm{u}, \mathrm{v})$

$I(u, v)=I_{b}(u, v)+I_{m}(u, v) \cos \left[\Delta \varphi(u, v)+\theta_{n}\right]$ 
, $n=1,2,3,4$

With steps of $\pi / 2$ the phase difference will take values of

$\theta_{n}=0, \pi / 2, \pi, 3 \pi / 2,2 \pi$

That is corresponding to path difference with step $\lambda / 4$, so a series of equation is produced as:

$I_{1}=I_{b}+I_{m} \cos (\Delta \varphi)$

$I_{2}=I_{b}-I_{m} \sin (\Delta \varphi)$

$I_{3}=I_{b}-I_{m} \cos (\Delta \varphi)$

$I_{4}=I_{b}+I_{m} \sin (\Delta \varphi)$

$I_{5}=I_{b}+I_{m} \cos (\Delta \varphi)$

The phase is then is derived -with some treatment- as

$\Delta \varphi=\tan ^{-1}\left(\frac{I_{2}-I_{4}}{I_{3}-I_{1}}\right)$

By using more steps it is possible to reduce the errors by several orders of magnitude but this five step bucket algorithm is sufficiently robust to the phase shift error since other errors are generally larger [8]

The measurement of step height using PSI is studied with the following parameters:

i- Vertical distance (z) between objective and sample.

ii- Intensity of the incident light.

iii- Angles of inclination of the sample to the incident light: roll and pitch angles (r) and (p).

iv- The numerical aperture of the used objective [5]

The step height measurement is significantly affected by wavefront curvature as the surface is considered as a single wavefront in PSI. The used light is a Gaussian filtered beam, with a wavefront curvature that is flipped when passing the focal plane as shown in figure (2) $[5,9]$.
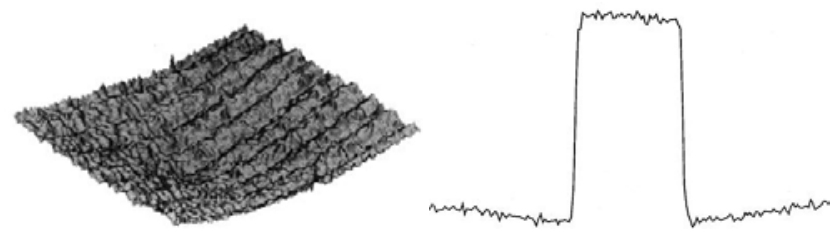

(a)
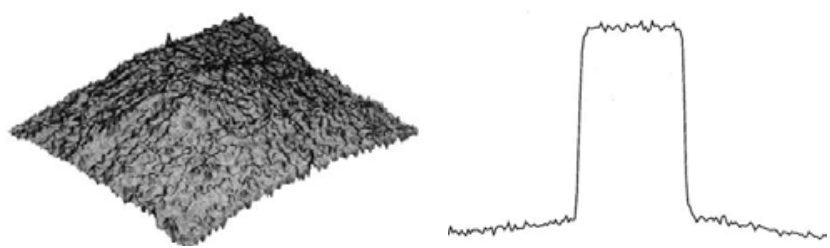

(b)

Figure (2) effect of wavefront defocus on the step height measurement using Gaussian filtered beam (a) Before focal plane, (b) after focal plane 
The intensity of used light affects the number of image pixels that are considered in surface parameter characterization, should neither be so low that a lot of data be missed due to darkness nor so high that a lot of data be missed due to light saturation.

The roll and pitch angle should be adjusted to guarantee the normal incidence of light onto the standard sample to obtain a full view of the sample surface, accordingly the vertical fringes should be parallel and of number three to four fringes [10]

\section{Numerical Aperture:}

the numerical aperture of an objective NA is defined as

$$
\mathrm{NA}=\mathrm{n} \sin \theta_{\max }
$$

where

$\mathrm{n}$ is the refractive index of the medium

$\theta_{\max }$ is the maximum incident accepted angle with which the light can physically reflect back into the objective lens and hence be measured as shown in figure(3)

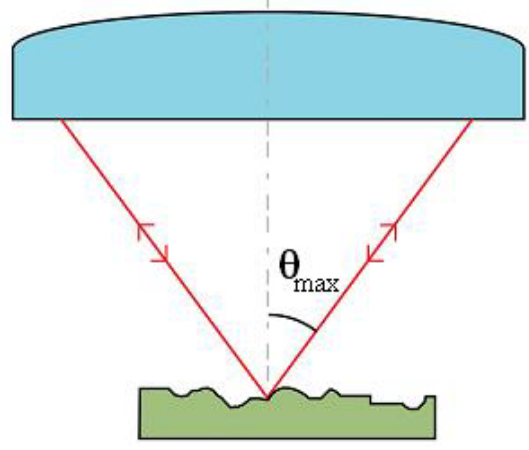

Figure (3) the maximum acceptance incident angle

\section{Experimental procedures}

A step height standard sample of nominal step height value h of $44 \mathrm{~nm}$ and 90.1 $\mathrm{nm}$ has been measured using the PSI mode with a filter to produce monochromatic light of $\lambda=633.4 \mathrm{~nm}$. The reflected intensity to the CCD camera was averaged five times in each run.. A 10X Mirau objective with Zygo interference microscope system NewView Maxim GP 200 enhanced with phase stepping facility and computerized stage. PSI technique with step height application has been used for the study of step height.

Step height application is one of Metropro applications library. The step height application algorithm uses the reference-test masking as shown in figure (4) where the sample area of interest is divided into reference (violet) mask and test (red) areas, each is averaged as least square plane of gathered points, the step height value is the net difference between reference and test masked area. 


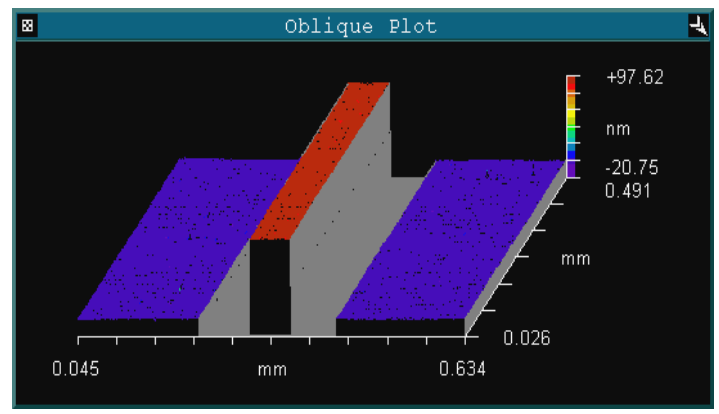

Figure (4) 3d plot of step height application

Study of step height with the vertical distance (z)

The vertical position of stage holding the standard was referenced at the maximum fringe contrast as the zero position then it is moved upward and downward to cover the vertical path of maximum coherence. The measurement is repeated five times at each $\mathrm{z}$ position.

Study of step height with the light intensity (I)

The vertical position of the stage was adjusted at the z-zero position as above then the height step is studied with the intensity of incident light.

Study of step height with the angles of inclination of the sample to the incident light: roll angle (r), the horizontal tilt and pitch angle (p), the vertical tilt the stage is initially adjusted at a position where $r$ and $p$ angles are small as possible that the fringes being vertical of about two fringes representing the total field of view, then the $r$ value is varied from -60 seconds to +60 seconds, while $p$ is varied from -200 to +200 to simulate the maximum probable human error in the tilt adjustment within an appropriate angular range for each angle

Study of step height with the numerical aperture (NA)

The study of numerical aperture of objectives is according to Chen [11] and the NA contribution to the step height measurement was calculated.

\section{System Noise:}

To estimate the system noise of the system, a silicon carbide optical flat reference of waviness of 1 Angstrom is used. The flat is measured twice at the same position with disabling any software option for frame averaging. The time difference between the successive runs is as short as possible. Once the first evaluated topography had accomplished, it would have been the subtraction error topography for the next one programmatically by a written Metroscript code. As this pair of runs is repeated 20 times, the results will represent the residual vibration of the system.

Study of internal reference plane:

The abovementioned flat reference is measured at sequential positions about 30 microns apart for each run, the larger the number of runs the closer flatness value from the reference plane flatness. 


\section{Results and discussion}

Step height with the vertical distance (h-z)

Figure (5) reveals a reversal proportional between step height values and the vertical position with respect to objective, which is in accordance with the wavefront curvature that leads to the discrepancy between step value where $\mathrm{z}$ before and after the focal plane as shown previously in figure 3 . The most reliable value will be at $\mathrm{z}=0$ where it is supposed to be planar wavefront, however the total variation in step height is $\Delta \mathrm{h}=4 \mathrm{~A}^{\circ}$.

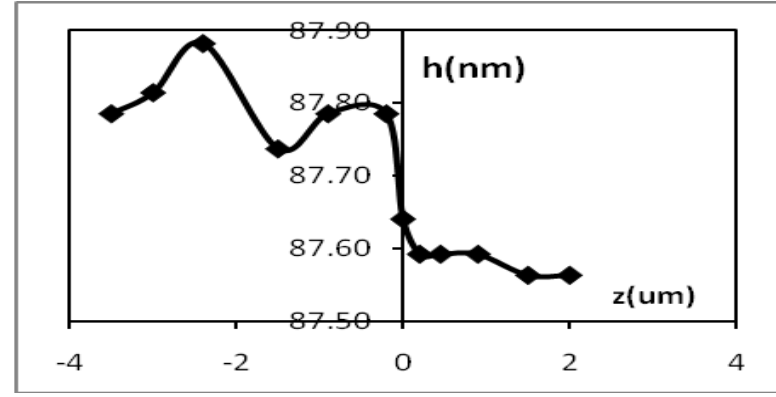

Fig.(5) step height study with the vertical distance apart the objective

Step height with the light intensity $(h-I)$

As shown in figure (6) the step height revealed no explicit trend has been detected with the light intensity that could be explained with the averaging effect of the gathered points in all masks least square technique of the step height algorithm. The selection of the incident light intensity appropriate to the selected objective's NA leads to least square plane at each masked area as mentioned above, so the loss of pixels due to more or less than the appropriate intensity has no great effect, about $1 \mathrm{~A}^{\mathrm{o}}$

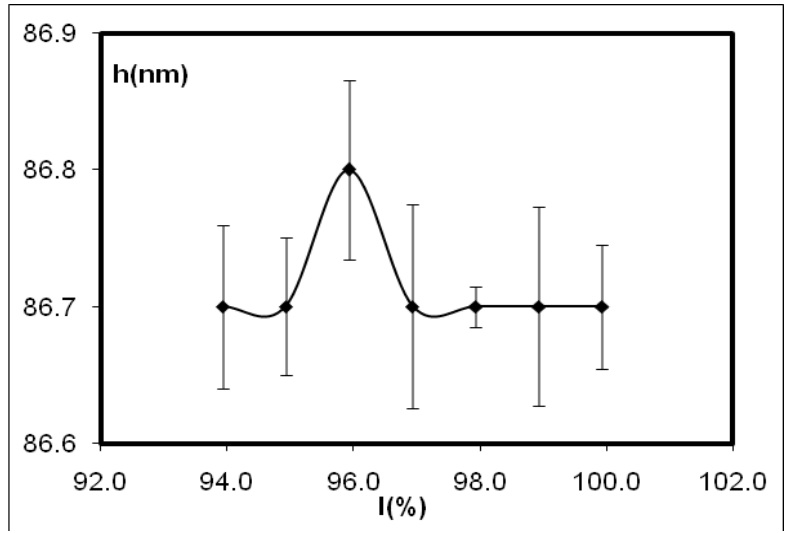

Fig.(6) the step height value has no clear trend with light intensity 
Step height with the tilt angles (h-r), (h-p)

It is found that the total contribution of the tilt angles ( $\mathrm{r}$ or roll angle in $\mathrm{X}$ direction and $\mathrm{p}$ or pitch angle in $\mathrm{Y}$ direction) to the step height value could be estimated with standard deviation about $0.09 \mathrm{~nm}$ for the nominal value of $90.1 \mathrm{~nm}$ using Mirau objective 50X, the error bars are of angstrom scale.

As shown in figure $7(a, b)$ the range of variation of tilt angle (r) is $120 \mathrm{sec}$ while in case of tilt angle (p) is $400 \mathrm{sec}$, that emphasizes the more influence of roll angle tilt direction that is perpendicular to the step edges, while the pitch angle tilt direction is parallel to the step. This is analogous to the tilt effect in stylus measurement of step height
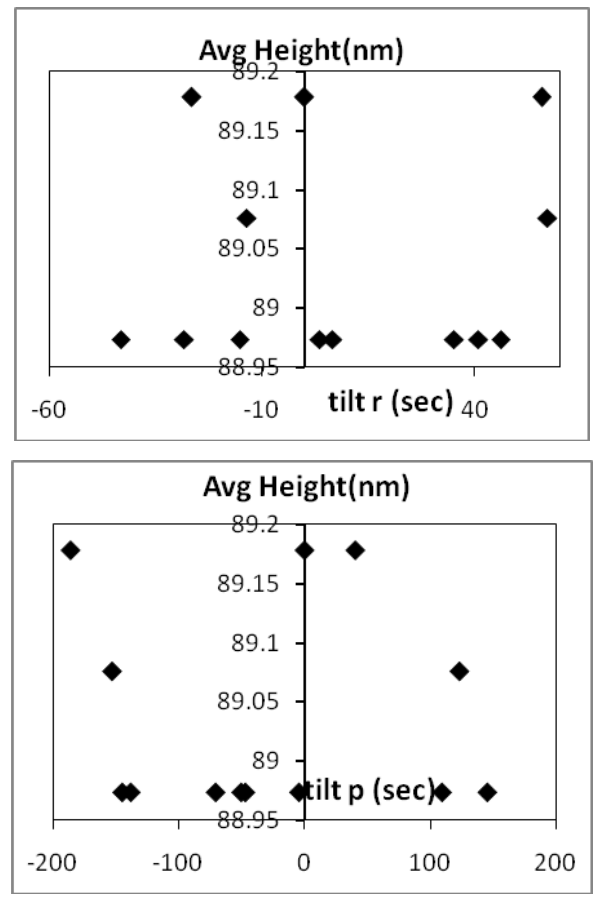

Fig.(7a,b) step height study with the roll and pitch tilting angle

Study of noise and flatness of reference plane noise $=0.2 \mathrm{~nm}[\sigma($ standard deviation $)=0.08 \mathrm{~nm}]$ flatness $=0.23 \mathrm{~nm}[\sigma($ standard deviation $)=0.1 \mathrm{~nm}]$

\section{Uncertainty budget according to the studied parameters}

The results were selected to represent the largest contribution of each studied parameter to the step height measurements using various objectives depending on the small variation of uncertainty between objectives are shown in table (1) according to GUM [12]. 
Table (1): The uncertainty budget according to the studied parameters

\begin{tabular}{|c|c|c|c|c|}
\hline source & $\begin{array}{c}\text { standard } \\
\text { uncertainty } \boldsymbol{u}_{i}\end{array}$ & $\begin{array}{c}v \\
\text { deg. of freedom }\end{array}$ & $\begin{array}{c}C_{\tilde{i}} \\
\text { Sensitivity } \\
\text { coeff. }\end{array}$ & $\begin{array}{l}\text { Compound uncertainty } \\
\qquad \frac{u_{i} C_{i}}{\sqrt{v-1}}\end{array}$ \\
\hline noise $\mathrm{nm}$ & 0.2 & 20 & 1 & 0.046 \\
\hline ref flatness $\mathrm{nm}$ & 0.23 & 16 & 1 & 0.059 \\
\hline wavefront curvature $\mathrm{nm}$ & 0.400 & 5 & 1 & 0.200 \\
\hline light intensity $\mathrm{nm}$ & 0.100 & 5 & 1 & 0.050 \\
\hline tilting of inc light $\mathrm{nm}$ & 0.09 & 5 & 1 & 0.045 \\
\hline NA correction factor & & & & 0.052 \\
\hline repeatability $\mathrm{nm}$ & 0.05 & 9 & 1 & 0.018 \\
\hline uc & \multicolumn{4}{|c|}{0.23} \\
\hline$U c=k^{*} u c$ & \multicolumn{4}{|c|}{0.46} \\
\hline
\end{tabular}

\section{References}

1. Gao, F., Muhamedsalih, Hussam and Jiang, Xiangqian, "Surface and thickness measurement of a transparent film using wavelength scanning interferometry", Optics Express, 20 (19). p. 21450. ISSN 1094-4087, 2012.

2. A. N. Korolev, V. I. Korotkov, A. Ya. Lukin, S. A. Pul'kin,, and A. L. Sizov, "Linear and angular measurements, measurement of the step height in the nanometric range using a laser microinterferometer” Measurement Techniques, Vol. 48, No. 4, 2005.

3. Matthew T. Rodgers, "Phase modulating interferometry with stroboscopic illumination for characterization of MEMS”, Master thesis, Worcester Polytechnic Institute , 2006.

4. Bengong Hao, Mingguang Shan, Zhi Zhong, Ming Diao, Yabin Zhang, "Common-path interferometer with four simultaneous phase-shifted interferograms using ronchi grating and cube beamsplitter”, Optics and Lasers in Engineering, 51 (1278-1282), 2013.

5. Takuma Doia, Theodore Vorburger, Paul Sullivanb "Effects of defocus and algorithm on optical step height calibration”, Precision Engineering 23(135-143) 1999

6. Kai Zhong, Zhongwei Li , Yusheng Shi, Congjun Wang, Yuzhen Lei, Optics and Lasers in Engineering,"Fast phase measurement profilometry for arbitrary shape objects without phase unwrapping", 51(1213-1222), 2013

7. James C. Wyant, "Advances in Interferometric Metrology", Optical Sciences Center, University of Arizona, Tucson, http://www.optics.arizona.edu/jcwyant

8. G. C. Brown, "Laser interferometric methodologies for characterizing static and dynamic behavior of microelectromechanical systems (MEMS)", Ph.D. Dissertation, Worcester Polytechnic Institute, Worcester, MA. 1999.

9. Andrew John Lewis, "Absolute length measurement using multiple-wavelength phasestepping interferometry", $\mathrm{PhD}$ thesis , University of London , 1993, reprinted 2002.

10. Richard Leach ,Leigh Brown, Xiangqian Jiang, "Measurement Good Practice Guide No. 108 Guide to the Measurement of Smooth Surface Topography using Coherence Scanning Interferometry”, NPL, 2008.

11. Yen-Liang Chen, Chao-Jung Chen, Chen-Yu Wang, "Determination of aperture correction factor of interference microscope by double-tilt imaging method", Center for Measurement Standards and Industrial Technology Research Institute, Taiwan, 2003

12. JCGM 100: 2008 GUM 1995 with minor corrections Evaluation of measurement data Guide to the expression of uncertainty in measurement , BIPM, 2008. 